INFLAMMATORY BOWEL DISEASE

\title{
Epithelioid granulomas, pattern recognition receptors, and phenotypes of Crohn's disease
}

\author{
M Pierik, G De Hertogh, S Vermeire, G Van Assche, P Van Eyken, S Joossens, G Claessens, \\ R Vlietinck, P Rutgeerts, K Geboes
}

Gut 2005;54:223-227. doi: 10.1136/gut.2004.042572

See end of article for authors' affiliations

Correspondence to:

Professor K Geboes,

Department of

Morphology and

Molecular Pathology,

University Hospitals

Leuven, Herestraat 49,

B-3000 Leuven, Belgium;

Karel.Geboes@

uz.kuleuven.ac.be

Revised version received 26 April 2004

Accepted for publication

11 May 2004

\begin{abstract}
Introduction: Crohn's disease is a chronic inflammatory disorder of the gut. It is assumed that a defective interaction between the bacterial flora of the gut and the innate immune system plays a key role in the pathogenesis of the disease. This may lead to specific histological lesions. The epithelioid granuloma is particularly interesting in this regard as it is also observed in several bacterial infections of the gut.

Aims and methods: We hypothesised that genetic or environmental factors with a known influence on inflammation or immunity would lead to an increased prevalence of granulomas. Therefore, surgical specimens from 161 patients were evaluated for the presence of granulomas. Patients were genotyped for the three single nucleotide polymorphisms in caspase recruitment domain 15 (CARD15)/NOD2 associated with CD and for Asp299Gly in Toll-like receptor 4 (TLR4).

Results: The overall prevalence of granulomas was $68.9 \%$. We did not find a significant correlation between granulomas and TLR4 or CARD1 5 variants. The frequency of granulomas increased with more distal disease (63\% small bowel, $72 \%$ right colon, $88 \%$ left colon, $90 \%$ rectum; $p=0.01$ ). Granulomas were more frequent in younger patients (odds ratio 0.95 (95\% confidence interval $0.92-0.98$ ) $p=0.007$ ). Conclusion: In this study of 161 well documented CD patients, we found no significant association between CARD 15 and TLR4 variants and granulomas. This finding seems to refute our initial hypothesis. However, it may be that additional factors are needed for granuloma development. Granulomas may develop only when specific bacterial components are present. Therefore, future research on granuloma pathogenesis should be orientated towards detection and identification of bacterial components in these lesions.
\end{abstract}

Abbreviations: $C D$, Crohn's disease; PRR, pattern recognition receptors; CARD, caspase recruitment domain; $L R R$, leucine rich repeat; SNP, single nucleotide polymorphism; TLR4, Toll-like receptor 4; ASCA, anti-Saccharomyces cerevisiae antibodies; $N F \kappa B$, nuclear factor $\kappa B$ impaired, possibly to
microbial components.

The major PRRs of the innate immune system are, however, the Toll-like receptors (TLRs). These receptors have an LRR in their extracellular domain. In particular, TLR2 and TLR4 have been shown to be essential for the recognition of distinct bacterial cell wall components. TLR2 discriminates peptidoglycan, lipoprotein, lipoarabinomannan, and zymosan whereas TLR4 recognises lipopolysaccharide, lipoteichoic acid, and Taxol. Bacterial components elicit activation of NFKB. ${ }^{9}$ It has been shown that the Asp299Gly mutation in the TLR4 receptor results in endotoxin hyporesponsiveness in humans. ${ }^{10}$ We have been able to associate this mutation with CD. ${ }^{11}$

Impaired recognition of bacterial components by the innate immune system may lead to specific histologically recognisable lesions. For instance, three missense mutations in the nucleotide binding domain of CARD15/NOD2 have been associated with Blau syndrome, a rare autosomal dominant disorder characterised by early onset granulomatous arthritis. ${ }^{12}$ Lesage et al found that Crohn's disease patients with double dose CARD15 mutations were characterised by younger age at onset, more frequent stricturing phenotype, and less frequent colonic involvement. There was also a borderline significant $(p=0.07)$ association between the presence of two mutations in CARD15/NOD2 and the development of granuloma in $\mathrm{CD} \cdot{ }^{13}$

A granuloma is a collection of macrophages and other inflammatory cells that surround a tenacious agent. Granuloma formation is normally a protective process by developing CD 2-4-fold whereas the presence of two mutations is associated with a 20-40-fold increase. The Leul007fsinsC SNP truncates NOD2 in the LRR region. Thus the LRR domain of CD associated variants is likely to be

- 
which noxious substances and infectious agents that resist rapid immune destruction are sequestered and prevented from damaging the surrounding tissue. The inflammation gradually destroys the irritants and clears the debris. Ultimately, scar tissue replaces the inflammation. Such a sequence is seen in tuberculosis and schistosomiasis. In schistosomiasis, infection is more intense and granulomas are more prevalent in younger individuals. ${ }^{14}$ Granulomas can also be seen in other examples of gut inflammation due to bacterial infection (for example, chlamydia, Yersinia pseudotuberculosis, salmonella, and campylobacter colitis). Epithelioid granulomas are among the most uniformly observed and specific microscopic features of $\mathrm{CD} .{ }^{15}$ However, the significance of epithelioid granulomas in CD is unclear. Several studies have attempted to identify an aetiological agent in such granulomas. The occasional finding of DNA specific for some microorganisms may represent either a bystander phenomenon or the cause of the disease. It is conceivable that particulate matter from dead organisms or very slow growing organisms can trigger the granulomatous reaction, although exacerbations of the disease are difficult to explain based on that hypothesis. Perhaps an autoimmune process intervenes. ${ }^{16}$

We hypothesised that mutations in CARD15 and TLR4 associated with CD might play a role in granuloma formation through over activation of the adaptive immune system in order to clear bacterial invasion. If this hypothesis is correct:

(1) there could be an association between CARD15 and TLR4 polymorphisms and the presence of epithelioid granulomas in surgical specimens of CD patients; and

(2) there could be a relation between the presence of epithelioid granulomas and certain phenotypic characteristics of CD patients (as seen in schistosomiasis where younger patients have more granulomas).

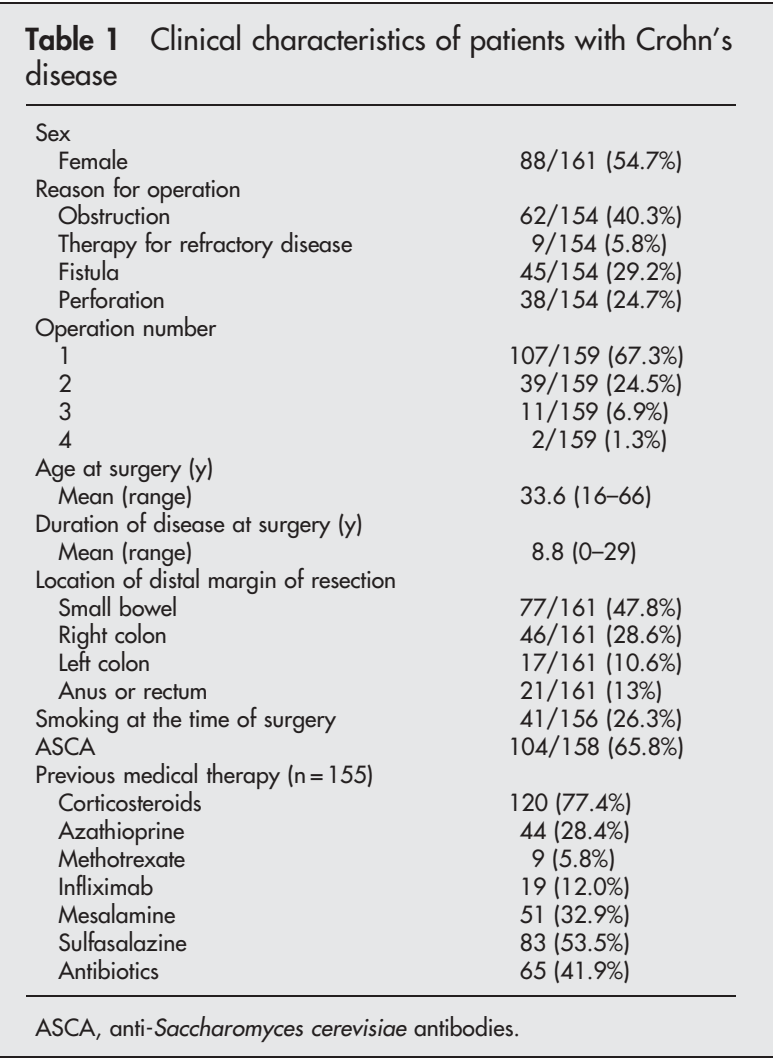

\section{MATERIALS AND METHODS}

\section{Patients}

We selected 161 CD patients from our IBD database. All subjects were surgically treated for $\mathrm{CD}$ at the University Hospital Gasthuisberg, Leuven, Belgium, between 1983 and 2003. Clinical charts were reviewed for the following characteristics: sex, smoking behaviour, ASCA status, indication for surgery (obstruction, perforation, fistulas, refractory disease), age and duration of disease at the time of surgery, previous medical treatment, operation number, and operation type (table 1). Venous blood was available for all patients. All subjects gave written informed consent and the ethics committee of the Katholieke Universiteit Leuven approved the study.

\section{Genotyping}

DNA was isolated from whole venous blood using a salting out procedure and was stored at $-80^{\circ} \mathrm{C}$. Patients were genotyped for the three main SNPs in CARD15 associated with CD (Arg702Trp, Gly908Arg, and Leul007InsC) and for the Asp299Gly SNP in TLR4 using polymerase chain reaction restriction fragment length polymorphisms, as described before. ${ }^{17} 18$ Primers and enzymes used are summarised in table 2. DNA restriction fragments were separated on agarose gels and visualised by ethidium bromide.

\section{Histology}

A total of 161 surgical specimens were selected for histological investigation. The size of the selected specimens ranged between 1 (anal lesions) and $91 \mathrm{~cm}$ (mean 31 (SD 16) $\mathrm{cm}$ ). Eighty per cent of the specimens were ileocolic resections or colectomies with a mean length of $32 \mathrm{~cm}$. Small bowel resections had a mean length of $29 \mathrm{~cm}$. For each patient, the location of the distal margin of the surgical specimen was noted (small bowel, right colon, left colon, rectum, or anus). In all specimens, the subserosal, mesenteric, or mesocolic fat was lamellated in slices of $5 \mathrm{~mm}$ in thickness. All obviously enlarged lymph nodes were sampled for histological examination. Enlarged lymph nodes were found in two thirds of the specimens. They were as frequent around the small bowel as pericolic. Up to 32 lymph nodes per specimen were examined. The median number of lymph nodes examined was five per specimen. The number of lymph nodes retrieved was independent of the type of resection specimen. The bowel wall was then opened longitudinally and the mucosal surface was inspected. Up to nine transmural biopsies were taken from macroscopically diseased and normal areas and snap frozen by immersion in liquid nitrogen. This material was stored at $-80^{\circ} \mathrm{C}$. Formalin fixed biopsies were processed for paraffin embedding. Sections ( $5 \mu \mathrm{m}$ thick) from all biopsies were stained with haematoxylin-eosin and examined for the presence of epithelioid granulomas. These were defined as discrete collections of at least five epithelioid cells (activated histiocytes with homogeneous eosinophilic cytoplasm) with or without accompanying multinucleate giant cells ${ }^{19}$ (fig 1). They were distinguished from isolated giant cells of foreign body type, microgranulomas, and pericryptal granulomas. ${ }^{20}$ Differential diagnosis against foreign body granulomas (defined by the presence of an identifiable foreign bodyfor example, food particles) was made. Such granulomas were rarely found and were excluded from subsequent analysis. Also excluded were granulomas bordering ulcer beds. The presence or absence of epithelioid granulomas was noted and their density was scored semiquantitatively (grade 1: no granulomas; grade 2: mucosal and/or submucosal granulomas or 1-10 granulomas present; and grade 3: granulomas in all bowel layers with or without granulomas 


\begin{tabular}{|c|c|c|c|}
\hline Gene & Variant & Primers & $\begin{array}{l}\text { Restriction } \\
\text { enzyme }\end{array}$ \\
\hline CARD15 & Arg702Trp & $\begin{array}{l}\text { 5'-AGATCACAGCAGCCTTCCTG-3' } \\
\text { 5'-CACGCTCTTGGCCTCACC- } 3^{\prime \prime}\end{array}$ & MSPI \\
\hline CARD15 & Gly908Arg & $\begin{array}{l}\text { 5'-CCCAGCTCCTCCCTCTTC-3' } \\
\text { 5'-AAGTCTGTAATGTAAAGCCAC-3' }\end{array}$ & Hhal \\
\hline CARD15 & Leu 1007/nsC & $\begin{array}{l}\text { 5'-GGCAGAAGCCCTCCTGCAGGGCC-3' } \\
\text { 5'-CCTCAAAATCTGCCATCC-3' }\end{array}$ & Apal \\
\hline TLR4 & Asp299Gly & $\begin{array}{l}\text { 5'-GATTAGCATACTTAGACTACTACCTCCATG-3' } \\
\text { 5'-GATCAACTTCTGAAAAAGCATTCCCAC-3' }\end{array}$ & $\mathrm{NCol}$ \\
\hline
\end{tabular}

in the mesenteric lymph nodes, or more than 10 granulomas present)..$^{15}$

\section{Statistics}

Genotypes and allele frequencies were compared between groups using the $\chi^{2}$ test or Fisher's exact test when appropriate (Statistica 6.0). The significance level was set at 0.05 . Logistic regression analysis applying a manual backward approach was used to define genetic and clinical variables in CD associated with granulomas (SAS 8.4).

\section{RESULTS}

\section{Histology}

The mean number of tissue blocks studied per resection specimen was 11 (SD 5). Epithelioid granulomas were present in the lymph nodes in $44 \%$ of patients where lymph nodes were investigated histologically. Granulomas were rarely seen in macroscopically normal bowel areas (in less than $10 \%$ of patients). When present, they were located in the mucosa. In macroscopically diseased areas, granulomas could be seen throughout the bowel wall.

Overall, epithelioid granulomas were present in $68.9 \%$ of patients. Thirty one per cent of patients had grade 1 (no granulomas), 9\% grade 2 (granulomas in the mucosa or submucosa or 1-10 granulomas present) and $60 \%$ grade 3 (granulomas in all bowel layers with or without those in lymph nodes, or more than 10 granulomas present). There were no clinical differences between the different granuloma grades except that patients with granulomas (grade 2 and 3 )

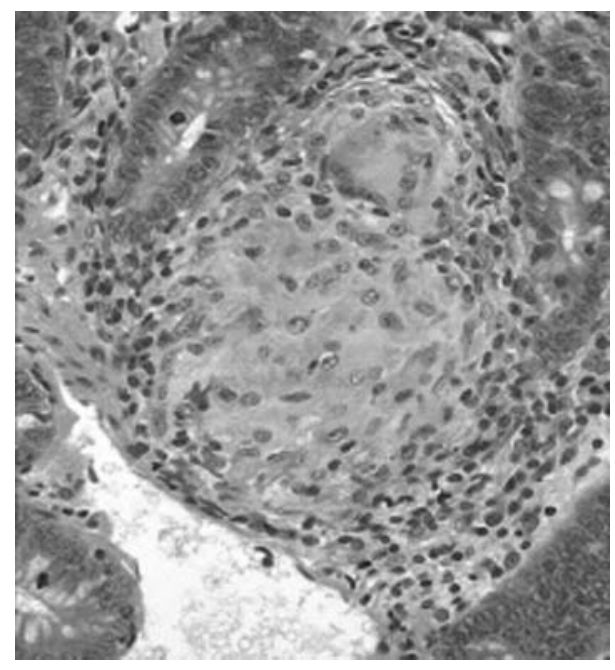

Figure 1 Epithelioid granuloma. Haematoxylin-eosin, original magnification $\times 400$.
Table 3 Logistic regression analyses of clinical variables associated with the presence of epithelioid granulomas (SAS 8.4 backward approach)

\begin{tabular}{|c|c|c|c|}
\hline & OR & $95 \% \mathrm{Cl}$ & p Value \\
\hline Age at operation & 0.95 & $0.92-0.98$ & 0.007 \\
\hline \multicolumn{4}{|c|}{ Most distal location of resection } \\
\hline Small bowel & 0.07 & $0.01-0.58$ & 0.01 \\
\hline Right colon & 0.16 & $0.02-1.43$ & - \\
\hline Left colon & 0.45 & $0.04-5.56$ & - \\
\hline Rectum/anus & Ref & Ref & Ref \\
\hline Smoking at time of operation & 2.45 & $0.95-6.66$ & 0.06 \\
\hline ASCA & 2.08 & $0.93-4.76$ & 0.08 \\
\hline
\end{tabular}

OR, odds ratio; $95 \% \mathrm{Cl}, 95 \%$ confidence interval.

ASCA, anti-Saccharomyces cerevisiae antibodies.

were younger $(p=0.0020)$ and were operated on earlier $(\mathrm{p}=0.0084)$ than patients without granulomas (grade 1$)$.

\section{Epithelioid granulomas, and CARD 15 and TLR4 polymorphisms}

Granulomas were present in 57/83 (68.7\%) patients without CARD15 mutations in comparison with 35/47 (74.5\%) heterozygous, $11 / 18(61.1 \%)$ compound heterozygous, and $6 / 11$ (54.5\%) homozygous patients. There was no significant difference in the prevalence of granulomas between these groups. We also did not find an association between CARD15 mutations and granulomas when the density of the granulomas was scored semiquantitatively.

Granulomas were present in 87/129(67.4\%) patients without TLR4 mutations and in 20/28 (71\%) patients with one or two TLR4 299Gly alleles. There was no significant difference in the prevalence of granulomas between these groups.

We further studied the combined effect of TLR4 and CARD15 mutations. Granulomas were present in 42/62 $(67.7 \%)$ patients without mutations, in 59/87 (67.8\%) patients with a mutation in either CARD15 or TLR4, and in $6 / 8(75 \%)$ patients with mutations in both genes. This also showed no difference.

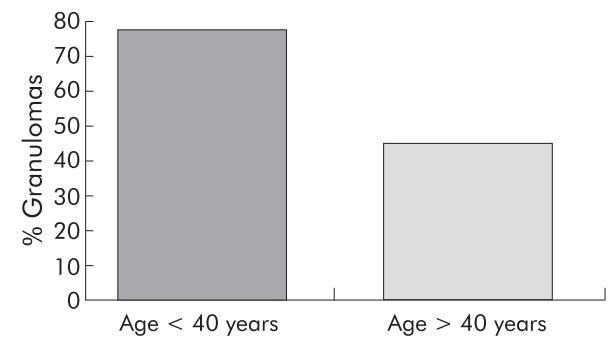

Figure 2 Presence of epithelioid granulomas and age at operation. 


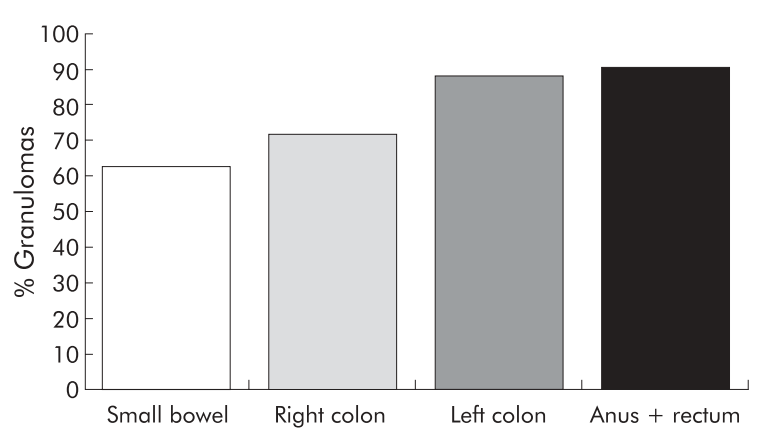

Figure 3 Presence of epithelioid granulomas and location of distal margin of resection specimen.

Epithelioid granulomas and clinical characteristics The logistic regression model reached an overall significance of $\mathrm{p}<0.001$ (table 3).

Patients with granulomas were younger when they underwent surgery (odds ratio (OR) 0.95 (95\% confidence interval (CI) $0.92-0.98) ; p=0.007$ ) (fig 2). The prevalence of granulomas increased significantly in more distal resections (small bowel, right colon, left colon, and rectum or anus had $62.8 \%, 71.7 \%, 88.2 \%$, and $90.4 \%$ granulomas, respectively; $\mathrm{p}=0.01)$ (fig 3). ASCA were more frequent in patients with granulomas (OR 2.08 (95\% CI 0.93-4.76); $\mathrm{p}=0.08$ ). Patients who were active smokers at the time of operation had granulomas more often than non-smokers (OR 2.45 (95\% CI $0.95-6.66) ; p=0.06$ ). No association between the prevalence of granulomas and sex, disease duration, number of operations, previous medical therapy, and indication for surgery was found.

\section{DISCUSSION}

Epithelioid granulomas in CD represent a chronic immune response to persistent, as yet unidentified, stimuli. We studied the association between granulomas and genetic and environmental factors implied in CD with a known influence on the inflammatory process or on the immune system. The studied variants in CARD15/NOD2 and TLR4 are functional and result in less NFKB activation after stimulation; thus it can be supposed that the innate immune response in the presence of these gene variants is impaired. We hypothesised that such impairment would be compensated for by over activation of the adaptive immune response. This over activation should manifest as a higher frequency of epithelioid granulomas in those CD patients with the variant CARD15 and TLR4 genes. However, we found no association between the presence of granulomas and CARD15 variants in 161 surgically treated CD patients. We also did not find an association between Asp299Gly in TLR4 and granulomas or between polymorphisms in the two receptors combined. These are probably valid results as they were obtained by study of a very large number of well documented patients.

It is known that the frequency of epithelioid granulomas in CD is extremely variable. The reported likelihood of finding these lesions varies from $15 \%$ to $82 \%$ of patients if surgical specimens are studied and from $3 \%$ to $56 \%$ of patients according to histological examination of endoscopic biopsies. ${ }^{21}$ In this study, the prevalence of surgical specimens was $68.9 \%$. We believe that this value is representative as we studied a large number of tissue blocks from many resection specimens.

Granulomas are also reported more frequently in younger patients. ${ }^{22}{ }^{23}$ In our study, patients who were younger at the time of surgery had granulomas more often. Thus we have confirmed in this large patient group the findings of
Heimann et al in their study of $44 \mathrm{CD}$ patients requiring surgery. ${ }^{23}$

A third finding was that the prevalence of granulomas was higher in resection specimens extending more distally. This result is similar to that described by Chambers and Morson in $1979^{24}$ and is related to the higher density of granulomas in the distal parts of the gastrointestinal tract. It can therefore be suggested that a higher amount of the causative agent is present distal in the bowel. The bacterial load of the gut is higher in the colon than in the ileum but the highest amount of bacteria is found in the caecum. No data on bacterial concentrations in the distal colon are available but bacterial diversity is highest distal in the colon. ${ }^{2}$

Antibodies against the baker's yeast ASCA are specific serological markers of CD and appear in $30-60 \%$ of patients. ${ }^{25}$ There is a familial appearance of ASCA and we previously described an association between CARD15 variants and ASCA. ${ }^{17}$ Lymphocytes and histiocytes in CD granulomas appeared to be stained specifically by biotinylated ASCA in a study by Oshitani and colleagues. ${ }^{26}$ They suggested that the altered immune response underlying CD results in impaired processing of baker's yeast, which possibly leads to granuloma formation. We observed a borderline significant higher prevalence of ASCA in patients with granulomas compared with patients without granulomas.

Smoking is a well known environmental risk factor for the development of CD. ${ }^{27} \mathrm{CD}$ patients who smoke have a higher relapse rate after surgery, they develop more severe lesions at the anastomotic site, and those who continue to smoke have a lower quality of life, ${ }^{28}$ suggesting that inflammation is more intense in CD patients who smoke. Although we found no significant association, we observed that our patients with granulomas smoked more often at the time of operation (29\%) than patients without granulomas $(20 \%)$.

\section{CONCLUSION}

In conclusion, we found no significant association between the patient's genotype for important CD associated CARD 15 and TLR4 variants and the presence of epithelioid granulomas in resection specimens. This result seems to refute our initial hypothesis. However, it may be possible that the hypothesis is correct but that additional factors are needed for granuloma development. It is plausible that granulomas develop only when specific bacterial components are present. Therefore, future research on granuloma pathogenesis should be orientated towards detection and identification of bacterial components in these lesions. Such an approach will probably also clarify why the density of granulomas increases in the distal gastrointestinal tract, and why these lesions never develop in a significant minority of patients.

\section{ACKNOWLEDGEMENTS}

This work was supported by a grant from the Funds for Scientific Research, Belgium, and from the National Crohn's and Colitis Organization (CCV) Belgium. MP is an FWO research fellow. SV is a postdoctoral FWO fellow.

\section{Authors' affiliations}

M Pierik*, S Vermeire, G Van Assche, S Joossens, G Claessens, P Rutgeerts, Department of Gastroenterology, University Hospitals, Katholieke Universiteit Leuven, Leuven, Belgium

G De Hertogh*, P Van Eyken, K Geboes, Department of Morphology and Molecular Pathology, University Hospitals, Katholieke Universiteit Leuven, Leuven, Belgium

R Vlietinck, Department of Epidemiology and Human Genetics, Katholieke Universiteit Leuven, Leuven, Belgium

*M Pierik and G De Hertogh contributed equally to this work. Conflict of interest: None declared. 


\section{REFERENCES}

1 Forbes A. Clinical presentation and diagnosis of Crohn's disease. In: Satsangi J, Sutherland LR, eds. Inflammatory bowel disease. Edinburgh: Churchill Livingstone, 2003:183-9.

2 Shanahan F. Host-flora interactions in inflammatory bowel disease. Inflam Bowel Dis 2004;10:S16-24.

3 Hugot JP, Chamaillard M, Zouali $\mathrm{H}$, et al. Association of NOD2 leucine-rich repeat variants with susceptibility to Crohn's disease. Nature 2001;411:437-9

4 Ogura Y, Bonen DK, Inohara N, et al. A frame shift mutation in NOD2 associated with susceptibility to Crohn's disease. Nature 2001;41 1:603-6.

5 Lala S, Ogura Y, Osborne C, et al. Crohn's disease and the NOD2 gene: a role for Paneth cells. Gastroenterology 2003;125:47-57.

6 Rosenstiel P, Fantini M, Brautigam K, et al. TNF-alpha and IFN-gamma regulate the expression of the NOD2 (CARD15) gene in human intestinal epithelial cells. Gastroenterology 2003;124:1001-9.

7 Hisamatsu T, Suzuki M, Reinecker HC, et al. CARD15/NOD2 functions as an antibacterial factor in human intestinal epithelial cells. Gastroenterology 2003; 124:993-1000

8 Inohara N, Ogura Y, Fontalba A, et al. Host recognition of bacterial muramyl dipeptide mediated through NOD2. Implications for Crohn's disease. J Biol Chem 2003;278:5509-12

9 Takeuchi O, Akira S. Toll-like receptors; their physiological role and signal transduction system. Int Immunopharmacol 2001;1:625-35.

10 Arbour NC, Lorenz E, Schutte B, et al. TLR4 mutations are associated with endotoxin hyporesponsiveness in humans. Nat Genet 2000;25:187-91.

11 Franchimont D, Vermeire S, El Housni H, et al. Deficient host bacteria interactions in inflammatory bowel disease: the Toll-like receptor (TLR)-4 Asp299Gly polymorphism is associated with Crohn's disease and ulcerative colitis. Gut 2004;53:987-92.

12 Miceli-Richard C, Lesage S, Rybojad M, et al. CARD15 mutations in Blau syndrome. Nat Genet $2001 ; 29: 19-20$.

13 Lesage S, Zouali H, Cézard and the EPWG-IBD group, et al. CARD14/NOD2 mutational analyses and genotype-phenotype correlation in 612 patients with inflammatory bowel disease. Am J Hum Genet 2002;70:845-57.

14 Ricosse JH, Emeric R, Courbil U. Anatomopathological aspects of schistosomiasis. A study of 286 pathological specimens (author's translation) Med Trop 1980;40:77-94.
15 Cook MG, Dixon MF. An analysis of the reliability of detection and diagnostic value of various pathological features in Crohn's disease and ulcerative colitis. Gut 1973;14:255-62

16 Mathew RC, Weinstock JV. Granuloma formation. In:Targan SR, Shanahan F, eds. Inflammatory bowel disease from bench to bedside. Philadelphia: Williams and Wilkins, 1994:151-9.

17 Esters N, Pierik M, Van Steen K, et al. Transmission of CARD15 (NOD2) variants within families of patients with inflammatory bowel disease. Am J Gastroenterol 2004;99:299-305.

18 Schmitt C, Humeny A, Becker CM, et al. Polymorphisms of TLR4: Rapid genotyping and reduced response to lipopolysaccharide of TLR4 mutant alleles. Clin Chem 2002;48:1661-7.

19 Jenkins D, Balsitis M, Gallivan S, et al. Guidelines for the initial biopsy diagnosis of suspected chronic idiopathic inflammatory bowel disease. The British Society of Gastroenterology Initiative. J Clin Pathol 1997; 50:93-105.

20 Shepherd NA. Granulomas in the diagnosis of intestinal Crohn's disease: a myth exploded? Histopathology 2002;4:166-8.

21 Heresbach D, Heresbach-Le Berre N, Ramee MP, et al. Fréquence et valeur pronostique du granulome épithélioïde au cours des maladies inflammatoires chroniques de l'intestin. Gastroenterol Clin Biol 1999;23:1376-87.

22 Schmitz-Moormann P, Pittner P, Sangmeister M. Probability of detecting granuloma in a colorectal biopsy of Crohn's disease. Pathol Res Pract 1984; 178:227-9

23 Heimann T, Miller F, Martinelli G, et al. Correlation of presence of granulomas with clinical and immunologic variables in Crohn's disease. Arch Surg 1988;123:46-8.

24 Chambers T, Morson BC. The granuloma in Crohn's disease. Gut 1979;20:269-74

25 Peeters M, Joossens S, Vermeire S, et al. Diagnostic value of antiSaccharomyces cerevisiae and antineutrophil cytoplasmic autoantibodies in inflammatory bowel disease. Am J Gastroenterol 2001;96:730-4.

26 Oshitani N, Hato F, Suzuki K, et al. Cross-reactivity of yeast antigens in human colon and peripheral leukocytes. J Pathol 2003;199:361-7.

27 Calkins BM. A meta-analysis of the role of smoking in inflammatory bowel disease. Dig Dis Sci 1989;34:1841-54.

28 Thomas G, Rhodes J, Green J, et al. Role of smoking in inflammatory bowel disease: implications for therapy. Postgrad Med J 2000;76:273-9.

\section{EDITOR'S QUIZ: GI SNAPSHOT}

\section{Answer}

From question on page 192

These alarming endoscopic pictures show acute oesophageal necrosis (AEN) or so-called black oesophagus. The criteria for diagnosis include the characteristic endoscopic appearance of a diffusely black oesophagus that always ends sharply at the gastrooesophageal mucosal junction. Ingestion of corrosive agents is excluded. AEN should be distinguished from other clinical entities, such as melanosis, pseudomelanosis, malignant melanoma, and acanthosis nigricans. AEN is confirmed histopathologically by diffuse necrosis. To our knowledge, to date only 30 cases have been reported in the literature, and the frequency was approximately $0.01 \%$ in patients undergoing upper endoscopy. AEN is often associated with deterioration of the general condition and the prognosis appears to depend mainly on the underlying illness. In this case, laboratory data revealed malnutrition and nephrotic syndrome. She was discharged, and follow up endoscopy showed an intact oesophageal mucosa three months later. When AEN is found, endoscopists should consider how to improve the patient's general condition.

doi: 10.1136/gut.2004.044784 